VP-5

\title{
Lap posterosuperior segmentectomy
}

\author{
Kyeong Deok KIM, Jong Man KIM*, Okjoo LEE, Sang Jin KIM, Manuel LIM, Ji Eun KWON, \\ Jaehun YANG, Eun Sung JEONG, Jinsoo RHU, Gyu-Seong CHOI, Jae-Won JOH \\ Department of Surgery, Samsung Medical Center, Seoul, Korea
}

Introduction: Surgical resection is one of the curative options for hepatocellular carcinoma (HCC). Posterosuperior segmentectomy is defined as anatomical resection of the liver segments 7 and 8 . However, minimally invasive resection of posterosuperior liver segments is considered a difficult and complex operation and is therefore rarely reported.

Methods: We present a video of laparoscopic posterosuperior segmentectomy of the liver $(\mathrm{S} 7,8)$ due to HCC, in a 66-years old male patient. Liver mobilization was performed with exposure of the inferior vena cava. Intraoperative ultrasound was used to locate the tumor and identify its relationship with the portal veins of segments 7 and 8 . The liver parenchyma was divided using ultrasonic energy device and the Glissonean pedicles were ligated using hem-o-lock and surgical clips. The surgical specimen was removed through a lower midline incision.

Results: Operative time was 162 minutes, and the estimated blood loss was $400 \mathrm{~mL}$. The patient recovered without problems such as admission to the intensive care unit or blood transfusion. Pathology confirmed progressed HCC.

Conclusions: Laparoscopic posterosuperior segmentectomy is difficult, but is feasible and safe. This video can help inexperienced surgeons perform this complex procedure. 